\title{
Thermal Efficiency of Laser Driven Inductively Coupled Plasma Generator Flows
}

\author{
By Makoto MAtsui $^{1)}$, Suisei YAmagishi ${ }^{2)}$, Kimiya Komurasaki ${ }^{3)}$, Yoshiki YAMAgIwA $^{1)}$ and Yoshihiro ARAKAwA ${ }^{2)}$ \\ ${ }^{1)}$ Department of Mechanical Engineering, Shizuoka University, Hamamatsu, Japan \\ ${ }^{2)}$ Department of Aeronautics and Astronautics, The University of Tokyo, Tokyo, Japan \\ ${ }^{3)}$ Department of Advanced Energy, The University of Tokyo, Kashiwa, Japan
}

(Received August 18th, 2009)

\begin{abstract}
A laser driven inductively coupled plasma generator was developed. Firstly, the radio frequency (RF) of $13.56 \mathrm{MHz}$ was applied to the atmospheric argon laser sustained plasma (LSP) in a quartz tube. As a result, the RF was successfully coupled to the LSP and following expansion of an emission region was observed. Next, the LSP was produced ahead of a ceramic convergent-divergent nozzle throat and the RF was applied from outside of the nozzle. The thermal efficiency estimated by a sonic flow method was ranged from $26 \%$ to $13 \%$.
\end{abstract}

Key Words: Laser Sustained Plasma, Inductively Coupled Plasma, High Enthalpy Flow

\section{Nomenclature}

$A^{*} \quad:$ Throat area

$C_{\mathrm{p}} \quad: \quad$ Specific heat at constant pressure

$D \quad$ : Defined in the text

$E \quad$ : Energy level of electronic excitation

$g \quad:$ statistical weight

$h \quad:$ Specific enthalpy

$k_{\mathrm{B}} \quad:$ Boltzmann constant

$\dot{m} \quad$ : Mass flow rate

$p \quad:$ Pressure

$P \quad: \quad$ Laser or RF power

$q \quad:$ Heat flux

$R^{*} \quad:$ Universal gas constant

$T \quad:$ Temperature

$\gamma \quad:$ Specific heat ratio

$\eta \quad:$ Thermal efficiency

Subscripts

$\begin{array}{ll}\text { amb } & : \text { Ambient } \\ \text { av } & : \text { Average } \\ \text { el } & : \text { Electronic excitation } \\ \mathrm{fr} & : \text { Forward } \\ i & : \text { Electronic state } \\ \text { laser } & : \text { Laser } \\ \text { max } & : \text { Maximum } \\ \mathrm{re} & : \text { Reflection } \\ \mathrm{s} & : \text { Stagnation } \\ \mathrm{tr} & : \text { Translational } \\ \mathrm{pl} & : \text { Plenum }\end{array}$

\section{Introduction}

Development of thermal protection systems (TPS) requires the simulation of entry conditions at ground test facilities. Typical entry conditions in past planetary exploration missions are tabulated in Table 1. Arc-heaters are powerful flow simulators to generate earth reentry conditions because of their long operational time, simple structure and ease of maintenance. ${ }^{1-4)}$ However, in studies of surface catalytic effects or active/passive oxidation of TPS materials, erosion of their electrodes has been recognized as an important obstacle because polluted flows make it difficult to evaluate chemical reaction rates in front of TPS surfaces. ${ }^{5-8)}$ In addition, carbon dioxide flows are difficult to operate because dissociated carbon deposits on the electrode surface, which causes undesirable short circuit. ${ }^{9)}$

On the other hand, inductively coupled plasma (ICP) generators have garnered much attention. ${ }^{10-13)}$ The ICP generators have no electrode. They can produce an ideal test condition for TPS tests because they have no undesirable chemical reactions that result from erosion. Another advantage of such generators is that they can use even reactive gases such as carbon dioxide and pure oxygen because of their electrode-less heating. However, in the ICP, plasma instability limits its operation pressure less than atmosphere. ${ }^{9)}$ Thereby, they can simulate Mars entry conditions whereas Venus conditions are still difficult to simulate.

Table 1. Entry conditions at peak heat flux.

\begin{tabular}{|c|c|c|c|c|}
\hline & Earth & Mars & Venus & Jupiter \\
\hline Atmosphere & $\mathrm{N}_{2}, \mathrm{O}_{2}$ & $\mathrm{CO}_{2}(95 \%)$ & $\mathrm{CO}_{2}(96 \%)$ & $\mathrm{H}_{2}, \mathrm{He}$ \\
\hline Mission & Apollo & $\mathrm{MPF}$ & Pioneer & Galileo \\
\hline$q_{\max }, \mathrm{kW} / \mathrm{cm}^{2}$ & 0.3 & 0.03 & 10 & 65 \\
\hline$p_{\mathrm{s}}$ at $q_{\max }, \mathrm{kPa}$ & 60 & 20 & 700 & 900 \\
\hline
\end{tabular}


Then, in order to simulate Venus entry conditions, we are developing a laser driven plasma wind tunnel. In this wind tunnel, test gases are heated through laser sustained plasma (LSP). ${ }^{14,15)}$ Since the LSP is produced by focusing a laser beam, it requires no-electrode and it is sustained even at the higher pressure than atmosphere. In our previous studies, ${ }^{16,17)}$ an argon-oxygen flow with the plenum pressure of $0.95 \mathrm{MPa}$ was successfully produced using a $2 \mathrm{~kW}$ class carbon dioxide laser. However, there are still some problems to be solved.

The first is a flow size. Since there is no laser comparable in power to arc-heaters and ICPs, it is difficult to produce the test flow which is large enough for TPS tests. Except for a military chemical laser, current maximum power of commercial continuous wave lasers is tens of kilo-watts. This value is one to two orders of magnitude lower than that of arc-heaters and ICPs currently in use.

The second is steep radial distributions of flow properties such as temperature, velocity, and specific enthalpy. ${ }^{16,17)}$ Since the core temperature of the LSP is estimated as 18,000 $\mathrm{K}$, ${ }^{18)}$ only a few percent of total working gas can path through the LSP core region due to a slightly high pressure region ahead of the LSP. ${ }^{14,15)}$ As a result, a main gas heating mechanism is not the laser absorption but the convection from the high temperature LSP to the surrounding gas. Then, flow properties have undesirable non-uniform radial distributions for TPS tests.

In order to solve these problems, the additional heating by the RF has been proposed. A combination of the RF would enable a future high power wind tunnel whose power level is not achieved only by lasers. Moreover, the additional annual heating by the RF might flatten the flow properties. In this study, the RF was applied to the LSP in a quartz tube to observe whether the RF was successfully coupled to the LSP. Next, to simulate a wind tunnel configuration, a ceramic convergent/divergent nozzle was developed. The LSP was produced ahead of the throat and the RF was applied from outside of the nozzle. Then, the thermal efficiency was measured by a sonic flow method.

\section{Laser Driven ICP Generator}

\subsection{Inductively coupled LSP}

A conceptual figure of the laser driven inductively coupled plasma generator is shown in Fig. 1. A laser beam is focused at a test gas in a plasma production chamber through a set of a condensing lens and a window. Once plasma is produced near the focal point, the plasma efficiently absorbs a beamed laser power through inverse bremsstrahlung radiation. This plasma is sustained where its propagation in the incident laser beam direction balances out the flow velocity. At the downstream of the LSP, the RF is applied using an induction coil. Although the ICP in general is difficult to be produced at the pressure higher than atmospheric pressure, it might be expected to be sustained using the LSP as a stationary electron source.

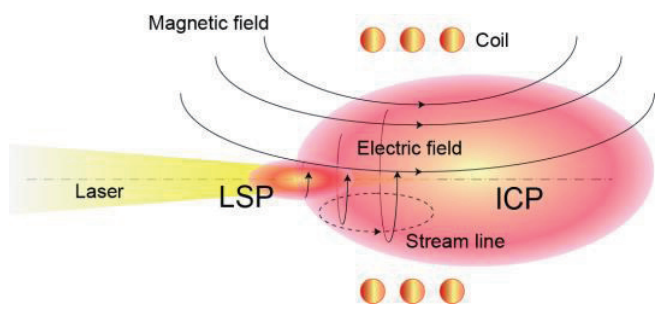

Fig. 1. Laser driven inductively coupled plasma generator.

\subsection{Experimental setup}

A schematic of a whole experimental setup is shown in Fig. 2. As a beam source, a continuous carbon dioxide laser (YB-L200B7T4, Matsushita Electric Industrial Co., Ltd.) is used. The wavelength is $10.6 \mu \mathrm{m}$. The maximum output power is $2 \mathrm{~kW}$ and the transverse mode of the laser beam is $\mathrm{TEM}_{10}$. The beam divergence angle is less than $2 \mathrm{mrad}$ at the laser exit. The beam diameter of $20 \mathrm{~mm}$ is magnified by 2.2 using a $\mathrm{ZnSe}$ beam expander and the expanded beam is condensed into the generator through a $\mathrm{ZnSe}$ plano-convex lens. The focal length of the lens is $250 \mathrm{~mm}$, corresponding to 7.4 in F-number which is defined as a ratio of the focal length to the beam diameter. This lens can move back to forth in the laser beam direction using a traverse stage. A metal rod made of stainless steel (SUS304) is used as the source of the initial electron emission. After the ignition, the LSP is moved into a quartz tube.

The quartz tube is $22 \mathrm{~mm}$ in inner diameter and $100 \mathrm{~mm}$ in length. The tube is supported by a set of water-cooled holders made of stainless steel. A water-cooled induction coil is made from $3 \mathrm{~mm}$ copper tube. It has an inside diameter of $30 \mathrm{~mm}$ and consists of 5 turns. The copper tube is covered with a thermal shrinkage tube to prevent from the short-circuiting between respective turns. A RF power generator with oscillation frequency of $13.56 \mathrm{MHz}$ and maximum output power of $1.25 \mathrm{~kW}$ (OWM-12, ENI) is utilized and connected to a matching circuit through $50 \Omega$ co-axial transmission line. The forward power and the reflected power are read out from the RF power supply. The matching circuit is composed of the load coil and two variable capacitors (T020-6066C, Thamway). The capacitance is manually adjusted to minimum the reflected power.

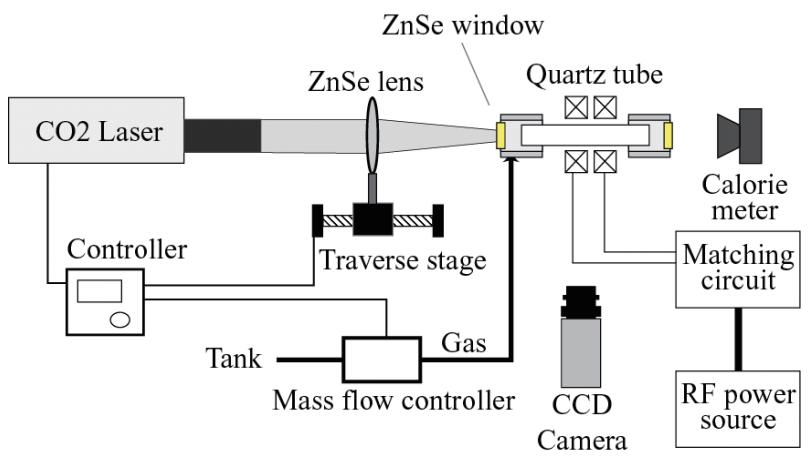

Fig. 2. Schematics of a whole experimental setup. 
Firstly, a double tube configuration is tested for plasma observation. The inner tube, whose inner diameter is $10 \mathrm{~mm}$, is expected to produce partially fast flow field in the center axis of the outer tube, as shown in Fig.3. ${ }^{19)}$ This fast flow field enables the LSP generation for a wide range of flow field parameters even if the RF is applied. Without the inner tube, the LSP moves upstream and sometimes disappears with the increase in the RF power.

Next, a convergent-divergent nozzle made of boron-nitride is set in a quartz tube assuming a future wind tunnel configuration as shown in Fig.4. The throat diameter is $2 \mathrm{~mm}$ and the nozzle exit diameter is $20 \mathrm{~mm}$. A heat-resisting ceramic bond is used to seal and connect the nozzle to the tube. The convergent part does not only choke the flow but also produce an acceleration flow field to stabilize the LSP.

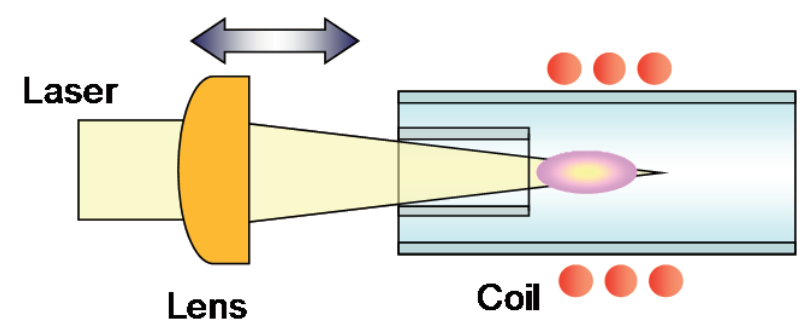

Fig. 3. Experimental setup for visualization of inductively coupled LSP.

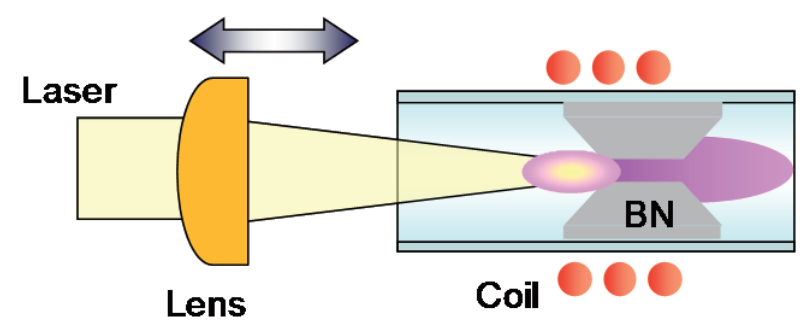

Fig. 4. Experimental setup for sonic flow method.

\section{Sonic Flow Methods}

In the ceramic nozzle experiment, the thermal efficiency is measured by a sonic flow method as follows. Assuming an isentropic flow, a quasi one dimension flow and a stationary flow, the averaged specific enthalpy is estimated expressed as, 20)

$$
h_{\mathrm{av}}=\left(\frac{A^{*} p_{\mathrm{pl}}}{\dot{m}}\right)^{2} \frac{\gamma^{2}}{\gamma-1}\left(\frac{2}{\gamma+1}\right)^{\frac{\gamma+1}{\gamma-1}} \text {. }
$$

Since argon is used as a working gas, a real gas effect on $C_{\mathrm{p}}$ is only electronic excitation expressed as, ${ }^{22)}$

$$
\begin{aligned}
C_{\mathrm{p}} & =C_{\mathrm{p}, \mathrm{tr}}+C_{\mathrm{p}, \mathrm{el}} \\
& =\frac{5}{2} R^{*}+\frac{R^{*}}{\left(k_{\mathrm{B}} T\right)^{2}}\left[\frac{D_{3}}{D_{1}}-\left(\frac{D_{2}}{D_{1}}\right)^{2}\right] .
\end{aligned}
$$

Here, $D_{1}, D_{2}, D_{3}$ are defined as,

$$
\begin{aligned}
& D_{1}=\sum_{i} g_{i} \exp \left(-E_{i} /\left(k_{\mathrm{B}} T\right)\right) \\
& D_{2}=\sum_{i} g_{i} E_{i} \exp \left(-E_{i} /\left(k_{\mathrm{B}} T\right)\right) . \\
& D_{3}=\sum_{i} g_{i} E_{i}^{2} \exp \left(-E_{i} /\left(k_{\mathrm{B}} T\right)\right)
\end{aligned}
$$

Figure 5 shows a contribution of the argon electronic excitation to $C_{\mathrm{p}}$ for a variation of the temperature. Since in our previous study, the throat temperature is less than $9000 \mathrm{~K}{ }^{17}$ ), $C_{\mathrm{p}, \text { el }}$ is negligible and Eq. (1) is valid. Then, the averaged specific enthalpy can be estimated by only measuring the plenum pressure.

The total thermal efficiency is estimated by the averaged specific enthalpy, the mass flow rate, the laser power and the net RF power expressed as,

$$
\eta=\frac{\dot{m} \cdot h_{\mathrm{av}}}{P_{\text {laser }}+\left(P_{\mathrm{fr}}-P_{\mathrm{re}}\right)} .
$$

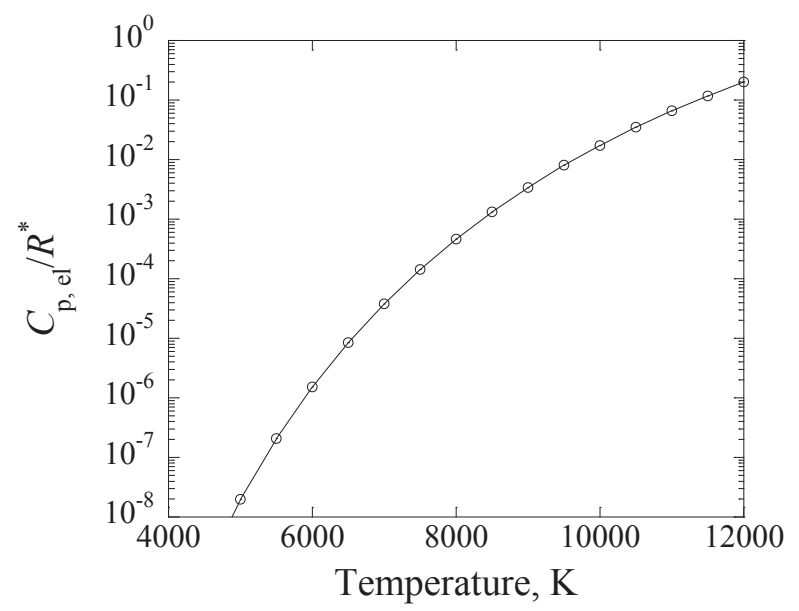

Fig. 5. Contribution of electronic excitation to $C_{\mathrm{p}}$.

\section{Results and Discussion}

\subsection{Inductively coupled LSP in a quartz tube}

Firstly, the RF was applied to the LSP in the single quartz tube to observe whether the RF was successfully coupled to the LSP. The laser and the RF generator were operated at 800 $\mathrm{W}$ and $770 \mathrm{~W}$, respectively. The mass flow rate of the argon was $1.03 \mathrm{~g} / \mathrm{s}$.

Figures 6 and 7 show photos of the LSP and the RF applied LSP, respectively. As seen in these figures, with the RF application, the plasma emission region was found to expand dramatically downstream of the LSP. The pressure was increased from $226 \mathrm{kPa}$ to $250 \mathrm{kPa}$ by the RF application. Then, it would appear that the RF was successfully coupled to the LSP.

An influence of the LSP position in the coil on the coupling efficiency was investigated. As a result, the reflected power was smallest when the LSP was set at the center of the induction coil. In this configuration, the reflection of the RF power was managed to be less than $3 \%$. 


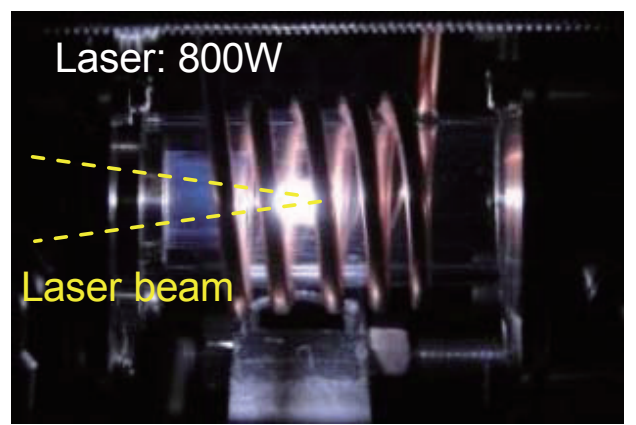

Fig. 6. Photo of LSP.

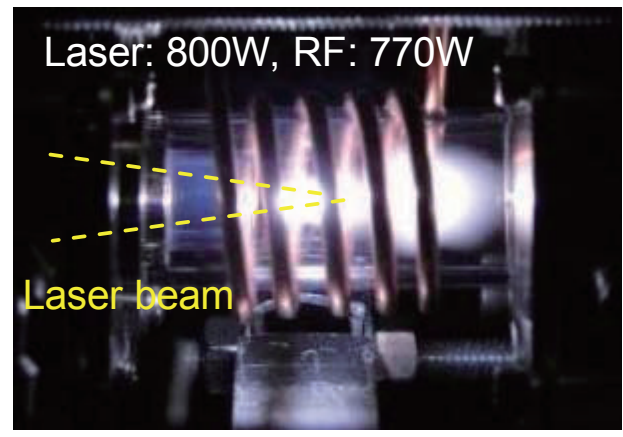

Fig. 7. Photo of inductively coupled LSP.

\subsection{Thermal efficiency measurements}

Next, the LSP was produced ahead of the ceramic nozzle throat and the RF was applied from outside of the nozzle. Judging by the result in a previous subsection, the throat position was set in the center of the induction coil to optimize the coupling efficiency. The thermal efficiency was estimated by the mass flow rate, the measured plenum pressure and the averaged specific enthalpy by the sonic flow method as described in the previous section. As operation conditions, for two cases of laser power of $700 \mathrm{~W}$ and $1000 \mathrm{~W}$, the RF generator was varied from $700 \mathrm{~W}$ to $0 \mathrm{~W}$ to $940 \mathrm{~W}$, respectively. Here, the RF power means net RF power defined by the input power minus reflected power. The argon mass flow rate was $1.49 \mathrm{~g} / \mathrm{s}$. The plenum pressure was $116 \mathrm{kPa}$ for the cold gas flow condition.

Figure 8 shows photos of the LSP and the RF applied LSP plumes. With the application of the RF, the plume emission region was found to be expanded in the axial and the radial direction. Figure 9 shows the thermal efficiency for a variation of the net RF power. The both thermal efficiencies decreased with the increase in the RF power. The efficiencies ranged from $26 \%$ to $13 \%$ for the $700 \mathrm{~W}$ LSP and $21 \%$ to $13 \%$ for the $1000 \mathrm{~W}$ LSP. These values are lower than those of conventional arc-heaters and ICPs. ${ }^{9,10)}$ This low efficiency might be because of the much larger coil diameter of $30 \mathrm{~mm}$ than the throat diameter of $2 \mathrm{~mm}$. In fact, the reflected RF power was more than $20 \%$ of the forward RF. Then, to enhance the thermal efficiency, a smaller diameter coil should be used and be set closer to the LSP. Another reason is that the RF might be coupled to the plume after the nozzle. Since the sonic flow method only takes into account the stagnation conditions upstream the throat, the electric power put into the plume is not counted. Then, the thermal efficiency may be underestimated.
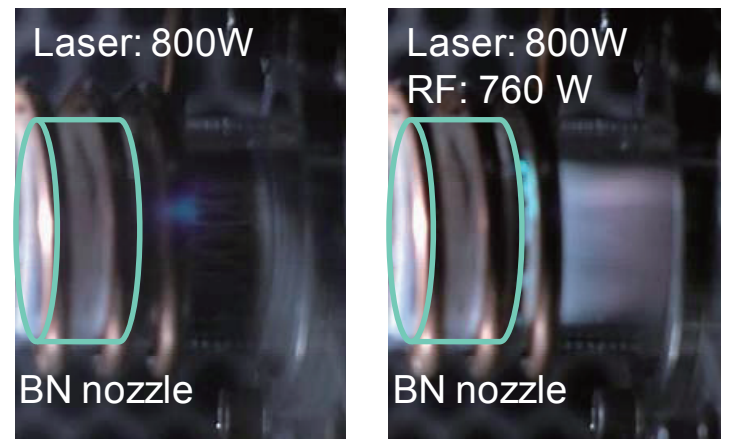

Fig. 8. Photos of the LSP plume (left) and the inductively coupled LSP plume (right).

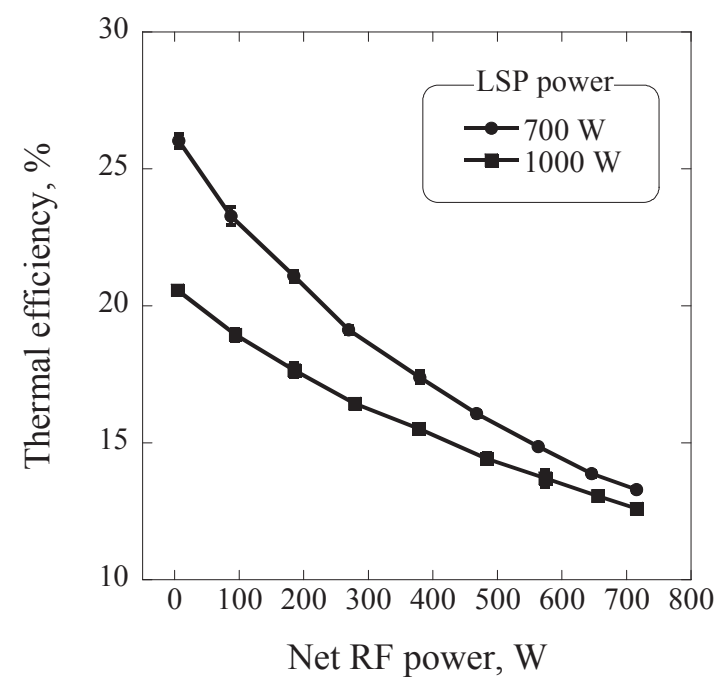

Fig. 9. Thermal efficiency for two different power LSPs.

\subsection{Comparison with LSP}

Figure 10 shows a comparison of the thermal efficiency between the LSP and the RF applied LSP. Here, the horizontal axis shows the additional laser power and the net RF power to the $700 \mathrm{~W}$ LSP. This means that the additional laser power of $700 \mathrm{~W}$ corresponds to the laser power of $1400 \mathrm{~W}$. As seen in this figure, the both efficiencies decreased with the increase in the additional input power. The efficiency in case of the RF application is a few percentages lower than that in the only LSP case. However, as discussed in the previous subsection, the thermal efficiency in case of the RF application might be underestimated and the coil configuration has room for improvement. Then, the combination of the RF with the LSP would be comparable to the LSP in the thermal efficiency. For more detail evaluation of this heating method, direct diagnostics of flow properties distributions in the plume are necessary. 


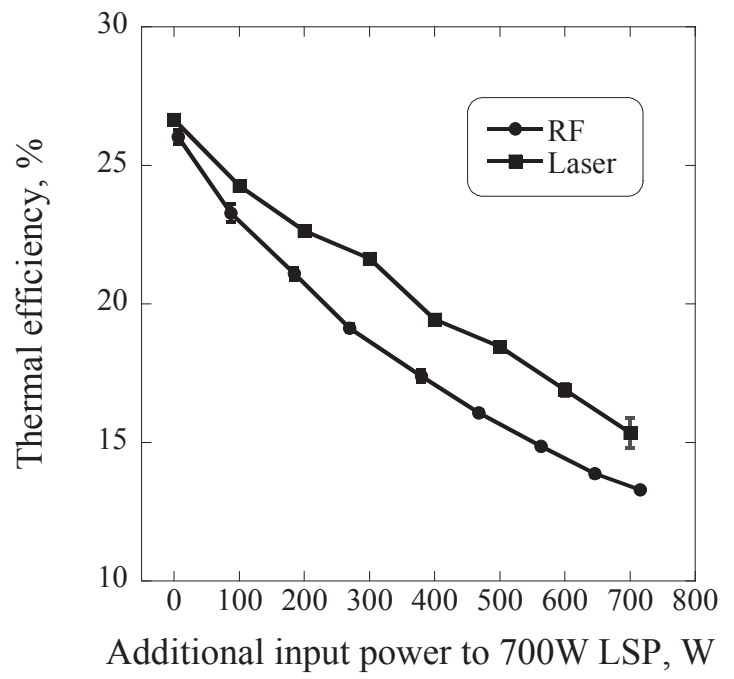

Fig. 10. Comparison of the thermal efficiency between LSP and RF applied LSP.

\section{Conclusion}

A laser driven inductively coupled plasma generator was developed. Firstly, the RF was applied to the atmospheric argon LSP in a quartz tube. As a result, the RF was successfully coupled to the LSP. It was found that the plenum pressure increased and the drastically expansion of the emission region was observed. The reflection was managed to be less than $3 \%$ when the LSP position was set at the center of the induction coil. Next, the LSP was produced ahead of a ceramic convergent divergent nozzle throat and the RF was applied from outside of the nozzle. For the variation of the net RF power from $0 \mathrm{~W}$ to $720 \mathrm{~W}$, the thermal efficiency estimated by a sonic flow method was $26 \%$ to $13 \%$ for the $700 \mathrm{~W}$ LSP, and $21 \%$ to $13 \%$ for the $1000 \mathrm{~W}$ LSP, respectively. These values were a few percentages lower than those of the only LSP case. However, the coil configuration has room for improvement. The coil size should be minimized as small as the throat size and closed to the LSP in order to enhance the thermal efficiency.

\section{Acknowledgments}

This research was partially supported by the Ministry of Education, Science, Sports and Culture, Grant-in-Aid for Exploratory Research, 16656263 and Research Fellowships of the Japan Society for the Promotion of Science for Young Scientists 18-09885.

\section{References}

1) Birkan, M. A.: Arcjets and Arc Heaters: An Overview of Research Status and Needs, J. Propulsion Power, 12 (1996), pp.1011-1017.

2) Auweter-Kurtz, M., Kurtz, H. L. and Laure, S.: Plasma Generators for Re-Entry Simulation, J. Propulsion Power, 12 (1996), pp.1053-1061.
3) Matsui, M., Takayanagi, H., Oda, Y., Komurasaki, K. and Arakawa, Y.: Performance of arcjet-type atomic-oxygen generator by laser absorption spectroscopy and CFD analysis, Vacuum, 73 (2004), pp.341-346.

4) Matsui, M., Ikemoto, T., Takayanagi, H., Komurasaki, K. and Arakawa, Y.: Generation of Highly Dissociated Oxygen Flows Using a Constrictor-Type Arc Heater, J. Thermophys. Heat. Tr., 21 (2007), pp.247-249.

5) Bykova, N. G., Vasil'evskii, S. A., Gordeev, A. N., Kolesnikov, A. F., Pershin, I. S. and Yakushin, M. I.: Determination of the Effective Probabilities of Catalytic Reactions on the Surfaces on Heat Shield Materials in Dissociated Carbon Dioxide Flows, J. Fluid Dynamics, 32 (1997), pp.876-886.

6) Balat, M., Flamant, G., Male, G. and Pichelin, G.: Active to Passive Transition in the Oxidation of Silicon Carbide at High Temperature and Low Pressure in Molecular and Atomic Oxygen, J. Materials Sci., 27 (1992), pp.697-703.

7) Kisa, M., Li, L., Yang, J., Minton, T., Stratton, W. G., Voyles, P., Chen, X., Benthem, K. and Pennyycook, S. J.: Homogeneous Silica Formed by the Oxidation of $\mathrm{Si}(100)$ in Hyperthermal Atomic Oxygen, J. Spacecraft Rockets, 43 (2006), pp.431-435.

8) Fujimoto, K, Satoh, K., Shioya, T., Seki, N. and Fujita, K. Degradation of Materials by High-Energy Atomic Oxygen, JSME International J. A, 46 (2002), pp.283-289.

9) Yamada, T., Fujita, K., Nonaka, S. and Ishii, N.: Performance Characteristics of the Inductively-Coupled High Enthalpy Flow Generator with $\mathrm{CO} 2 \mathrm{Gas}$, Proceedings of $25^{\text {th }}$ International Symposium on Space Technology and Science, 2006, e-19.

10) Herdrich, G. and Auweter-Kurtz, M.: Development and Characterization of Inductively Heated Plasma Generator for Atmospheric Entry Simulations, AIAA Paper 04-2503, 2004.

11) Matsui, M, Komurasaki, K., Herdrich, G. and Auweter-Kurtz, M.: Enthalpy Measurement in Inductively Heated Plasma Generator Flow by Laser Absorption Spectroscopy, AIAA J. 43 (2005), pp.2060-2064

12) Matsui, M., Komurasaki, K., Arakawa, Y., Knapp, A., Herdrich, G. and Auweter-Kurtz, M.: Enthalpy Measurement of Inductively Heated Air Flow, J. Spacecraft Rockets, 45 (2008), pp.155-157.

13) Ito, I., Ishida, K., Mizuno, M., Sumi, T., Fujita, K., Nagai, J., Murata, H. and Matsuzaki, T.: Heating Tests of TPS samples in $110 \mathrm{~kW}$ ICP-heating wind tunnel, Proceedings of $24^{\text {th }}$ International Symposium on Space Technology and Science, 2004, e-20.

14) Toyoda, K. Komurasaki, K. and Arakawa, Y.: Thrust Performance of a CW Laser Thruster in Vacuum, Vacuum, 65 (2002), pp.383-388.

15) Inoue, T., Uehara, S., Komurasaki, K. and Arakawa, Y.: Energy Conversion in a CW Laser Thruster, J Jpn. Soci. Aeronaut. Space Sci., 54 (2006), pp.168-174.

16) Matsui, M., Shinmi, K., Ueno, T., Komurasaki, K. and Arakawa, Y.: Operation Characteristics of Laser Driven Plasma Wind Tunnel, Trans. JSASS Space Tech. Japan, 7 (2009), pp.31-36.

17) Matsui, M., Shinmi, K., Komurasaki, K. and Arakawa, Y.: Enthalpy Distributions of Laser Driven High Enthalpy Wind Tunnel, AIAA Paper 08-4133, 2008.

18) Hosoda, S., Inoue, T., Komurasaki, K. and Arakawa, Y.: Study of Energy Conversion Process in CW laser Propulsion, Proceedings of $28^{\text {th }}$ International Symposium on Electric Propulsion Conference, 2003, 03-198.

19) Inoue, T., Uehara, S., Komurasaki, K. and Arakawa, Y.: Inductively Coupled Plasmas Supported by Laser Plasmas for High Enthalpy Flow, AIAA Paper05-110, 2005.

20) Winovich, W.: On the Equilibrium Sonic-Flow Method for Evaluating Electric-Arc Air-Heater Performance, NASA TN D-2132, 1964.

21) Bose, T. K.: High Temperature Gas Dynamics, Springer, Berlin, 2004. 